\section{The Effect of Probiotic Supplementation during Pregnancy and Lactation on Infantile Atopic Dermatitis}

\author{
Abbreviations \\ AD: Atopic Dermatitis; RCT: Randomized Control Trial; \\ OTC: Over-The-Counter; LPR: Lactobacillus rhamnosus; ST111: \\ Lactobacillus paracasei; BL999: Bifidobacterium longum; cfu: Colony \\ Forming Units
}

Due in part to the substantial increase in the number of cases of pediatric atopic dermatitis (AD) cases over the last three decades in the developing world [1], there is a trend towards approaching therapy for $\mathrm{AD}$ using natural therapies. An increasingly common question that dermatologists encounter from parents is whether or not probiotic supplementation during pregnancy and while breastfeeding reduces the risk of atopy and pediatric AD. The following commentary addresses this knowledge practice gap by discussing whether probiotic supplementation can treat or prevent pediatric $\mathrm{AD}$.

Probiotics play an important role in immunological, digestive, and respiratory functions and may reduce infectious disease in children [1]. The consensus of a meta-analysis of 21 studies, including infants and mothers who were pregnant or breastfeeding and supplemented with probiotics, revealed probiotic supplementation was effective in preventing (in 11 of 17 studies) or decreasing severity of (in 5 of 6 studies) pediatric $\mathrm{AD}$ [2]. In two studies that failed to demonstrate the preventative effect of probiotics, one study did not provide the mothers with perinatal supplementation during the last four weeks of pregnancy [3], and another failed to directly supplement the child during the first three months of life [4]. The first three months of life represent a critical period of immune maturation [5] and suggest that direct supplementation to the baby is more important than receiving passive exposure through breast milk.

Probiotics are not universally accepted as a preventative measure. In a recent study by Abrahammsson et al, probiotics were not seen to have a definitive preventative effect against AD [5]. Nonetheless, infants who received probiotics had less IgE-associated eczema at 2 years of age than those who did not. Skin prick test reactivity also decreased in the treatment group but was only a statistically significant finding among infants with allergic mothers $[2,6]$. However, mothers with a history of atopy may be more likely to ask about possible therapies than those without these problems.

Administering viable bacteria to a newborn infant has also raised safety and contamination concerns since over the counter (OTC) probiotics are not FDA regulated. However, previous investigation into the safety and efficacy of perinatal probiotic supplementation has shown that probiotics are safe and can reduce $\mathrm{AD}$ in high-risk infants

\section{Clinical \& Investigative Dermatology}

\author{
Collin M Blattner ${ }^{1}$ and Jenny E Murase ${ }^{2,3 *}$ \\ ${ }^{1}$ Des Moines University, IA, USA \\ ${ }^{2}$ Department of Dermatology, University of California, San Francisco, \\ San Francisco, CA, USA \\ ${ }^{3}$ Department of Dermatology, Palo Alto Foundation Medical Group, \\ Mountain View, CA, USA
}

\section{*Address for Correspondence}

Jenny E. Murase, Department of Dermatology, Palo Alto Foundation Medical Group, 701 East El Camino Real (31-104), Mountain View, CA 94040, Tel: 650-934-7676; Fax: 650-934-7696; E-mail: jemurase@gmail.com

Submission: 20 December 2013

Accepted: 05 March 2014

Published: 10 March 2014

[7]. In a randomized control trial (RCT) of 241 mother-infant pairs, maternal probiotic supplementation during the last two months of pregnancy and the first two months of breastfeeding significantly reduced the risk of eczema during the first two years of life in comparison to infants whose mothers received placebo [7]. This was further substantiated by another high-risk birth cohort study where maternal supplementation from 35 weeks gestation until 6 months post-gestation with breastfeeding and infant supplementation from birth until 2 years with Lactobacillus rhamnosus (LPR) halved the cumulative prevalence of eczema at 2 and 4 years [8].

Gastrointestinal symptoms were the most common side effect and were slightly more common among mother-infant pairs who received probiotics. Of the women who experienced side effects, 4/70 (6\%) received placebo, 6/80 (8\%) received LPR with Bifidobacterium longum (BL999), and 3/74 (4\%) received Lactobacillus paracasei (ST111) with BL999 [7]. Gastrointestinal symptoms were observed in 87/223 (39\%) of the infants when probiotics were administered to breastfeeding mothers [6]. The rates of gastrointestinal symptoms among infants whose mothers received placebo, LPR1BL999, or ST111BL999 were $24 / 70$ (34\%), $35 / 79$ (44\%), and 28/74 (38\%), respectively [7]. This suggests that maternal probiotic supplementation is safe in infants with high hereditary risk for atopy. If still concerned, one may administer probiotics to the mother only, which will further increase the safety profile as noted in studies in Norway [9] and Korea [10].

LPR currently has the most data to support long-term prevention of $\mathrm{AD}$ development [2]. Dermatologists should recommend the use of OTC LPR to achieve the most significant effect since this probiotic strain is not available in prescription form. The only prescription probiotic available is VSL \#3-DS, which has been deemed safe by the Federal Drug Administration. LPR is manufactured by the same company that makes VSL \#3-DS and contains more live bacteria then non-prescription VSL\#3.

Therefore, if you have a patient who is planning to use a probiotic, encourage her to take LPR two months prior to her due date. Also, advise the mother to take the supplement when breastfeeding and give the supplement directly to her baby after birth. The recommended capsular dose for both mother and infant is $6 \times 10^{9}$ colony forming units; however, the mother should break up the contents and dilute 
Citation: Blattner CM, Murase JE. The Effect of Probiotic Supplementation during Pregnancy and Lactation on Infantile Atopic Dermatitis. J Clin Investigat Dermatol. 2014;2(1): 2.

in a small glass of water for the infant [11]. Probiotics are safe, relatively effective, and may reduce the risk of her child developing $\mathrm{AD}$ later in life. However, we would discourage use of probiotics as the sole treatment modality for pediatric $\mathrm{AD}$ given the importance of emolliation, good skin care practices, and topical anti-inflammatory therapies to control the condition.

\section{References}

1. Andersson H, Asp NG, Bruce $\AA$, Roos S, Wadström T, et al.(2001) "Health effects of probiotics and prebiotics A literature review on human studies." Food \& Nutrition Research 45: 58-75.

2. Foolad N, Brezinski EA, Chase EP, Armstrong AW (2013) Effect of nutrien supplementation on atopic dermatitis in children: a systematic review of probiotics, prebiotics, formula, and fatty acids. JAMA Dermatol 149: 350-355.

3. Taylor AL, Dunstan JA, Prescott SL (2007) Probiotic supplementation for the first 6 months of life fails to reduce the risk of atopic dermatitis and increases the risk of allergen sensitization in high-risk children: a randomized controlled trial. J Allergy Clin Immunol 119: 184-191.

4. Kopp MV, Hennemuth I, Heinzmann A, Urbanek R (2008) Randomized double-blind, placebo-controlled trial of probiotics for primary prevention: no clinical effects of Lactobacillus GG supplementation. Pediatrics 121: e850856.
5. Rautava S, Arvilommi H, Isolauri E (2006) Specific probiotics in enhancing maturation of IgA responses in formula-fed infants. Pediatr Res 60: 221-224.

6. Abrahamsson TR, Jakobsson T, Böttcher MF, Fredrikson M, Jenmalm MC, et al. (2007) Probiotics in prevention of IgE-associated eczema: a double-blind, randomized, placebo-controlled trial. J Allergy Clin Immunol 119: 1174-1180.

7. Rautava S, Kainonen E, Salminen S, Isolauri E (2012) Maternal probiotic supplementation during pregnancy and breast-feeding reduces the risk of eczema in the infant. J Allergy Clin Immunol 130: 1355-1360.

8. Wickens K, Stanley TV, Mitchell EA, Barthow C, Fitzharris P, et al. (2013) Early supplementation with Lactobacillus rhamnosus HN001 reduces eczema prevalence to 6 years: does it also reduce atopic sensitization?. Clinical \& Experimental Allergy 43: 1048-1057.

9. Dotterud CK, Storro O, Johnsen R, Oien T (2010) Probiotics in pregnant women to prevent allergic disease: a randomized, double-blind trial. $\mathrm{Br} \mathrm{J}$ Dermatol 163: 616-623.

10. Kim JY, Kwon JH, Ahn SH, Lee SI, Han YS, et al. (2010) Effect of probiotic mix (Bifidobacterium bifidum, Bifidobacterium lactis, Lactobacillus acidophilus) in the primary prevention of eczema: a double-blind, randomized, placebocontrolled trial. Pediatr Allergy Immunol 21: e386-393.

11. Kalliomäki M, Salminen S, Arvilommi H, Kero P, Koskinen P, et al. (2001) Probiotics in primary prevention of atopic disease: a randomized placebocontrolled trial. Lancet 357: 1076-1079.

\section{Copyright}

(C) 2014 Blattner CM, et al. This is an open access article distributed under the Creative Commons Attribution License, which permits unrestricted use, distribution, and reproduction in any medium, provided the original work is properly cited. 